\title{
A protective role for microRNA-688 in acute kidney injury
}

\author{
Nicholas Chun, ${ }^{1}$ Steven G. Coca, ${ }^{1}$ and John Cijiang He ${ }^{1,2}$ \\ 'Department of Medicine/Nephrology, Icahn School of Medicine at Mount Sinai, New York, New York, USA. ${ }^{2}$ Renal Section, James J. Peters Veterans Affair Medical Center, New York, New York, USA.
}

\begin{abstract}
Ischemia-reperfusion (I/R) sets off a devastating cascade of events, leading to cell death and possible organ failure. Treatments to limit I/R-associated damage are lacking, and the pathways that drive injury are poorly understood. In this issue of the $J C l$, Wei and colleagues identify microRNA-668 (miR-668) as a protective factor in acute kidney injury (AKI). miR-668 was shown to repress mitochondrial fissionassociated protein MTP18, thereby inhibiting pathogenic mitochondrial fragmentation. In murine models of I/R-induced AKI, treatment with a miR-668 mimetic reduced mitochondrial fragmentation and improved renal function. Moreover, HIF-1 $\alpha$ was shown to be required for miR-688 expression in response to I/R. Importantly, Wei et al. show miR-668 upregulation in a cohort of human patients with AKI. Together, these results identify a HIF-1 $\alpha / \mathrm{miR}-668 / \mathrm{MTP} 18$ axis that may have potential as a therapeutic target for AKI.
\end{abstract}

as cytochrome $c(3,16,17)$, and/or the activation of pronecroptotic proteins such as RIP1 kinase (9).

More recently, epigenetic mechanisms, including the upregulation of a diverse array of tissue-specific protective and pathogenic microRNAs (miRs), have been described in the setting of I/R (18). miRs regulate gene expression by preventing translation or inducing degradation of target-gene mRNAs, and several miRs have been mechanistically linked to I/R injury $(3,18,19)$. In this issue, Wei et al. determine that miR-668, previously found to be upregulated in a murine model of renal $\mathrm{I} / \mathrm{R}$ injury (19), is protective during renal I/R by inhibiting pathogenic mitochondrial fragmentation and consequent renal tubular cell apoptosis (20).

\section{Ischemia-reperfusion and acute kidney injury}

Ischemia-reperfusion (I/R) injury occurs broadly across organ systems, causing substantial human disease; however, effective treatments are lacking. Clinically, I/R manifests as heart attack, stroke, ischemic hepatitis/bowel, lung ischemia, acute kidney injury (AKI), and posttransplant allograft dysfunction (1-5). Sensitivity to ischemia is variable and organ specific. Moreover, in the kidney, there are also regional differences, with proximal tubules more susceptible to ischemia than the inner medulla and deep papillae (2).

The cellular mechanisms of I/R injury are diverse and incompletely understood. Current concepts are fully reviewed elsewhere $(1-3,6)$ and implicate a cascade of interacting events, including calcium overload, mitochondrial dysfunction, generation of reactive oxygen species (ROS), endoplasmic reticulum (ER) stress, and induction of inflammation, that are initiated during ischemia and paradoxically exacerbated after reperfusion. Cumulatively, these pathogenic events induce apoptotic $(7,8)$ or necrotic (9) cell death via cell-intrinsic and/or I/R-induced inflammatory mechanisms. Components of innate immunity, such as complement, the inflammasome, and neutrophils, and the adaptive immune system, including $\mathrm{T}$ cells, have been implicated in I/R injury of both native and transplanted organs (10-15). The intrinsic mechanisms implicated in I/R-associated cell death include disruption of mitochondrial dynamics, resulting in mitochondrial fragmentation, the release of proapoptotic proteins such

\section{Related Article: p. 5448}

Conflict of interest: SGC is a cofounder of RenalytixAI and SGC and JCH are members of the advisory board of Renalytix$\mathrm{Al}$ and own equity in the same. JCH received research support from Shangpharma Innovation and has a pending patent on "Oxadiazole and Thiadiazole Inhibitors of HIPK2 for Treating Kidney Fibrosis." SCC has received consulting fees from Goldfinch Bio, CHF Solutions, Quark Biopharma, Janssen Pharmaceuticals, and Takeda Pharmaceuticals. SCC has a patent pending ["Compositions and Methods for Assessing Acute Kidney Injury (AKI) and Mortality Risk"].

Reference information: / Clin Invest. 2018;128(12):5216-5218. https://doi.org/10.1172/JCI124923.

\section{A protective miR-668- mediated axis}

Wei et al. found that inhibition of miR668 with an anti-miR-668 locked nucleic acid (LNA) worsened I/R-associated AKI in a murine model, as measured by blood urea nitrogen (BUN) and serum creatinine levels 48 hours after ischemic insult. There were no appreciable histologic differences in the kidneys between control and LNA-treated mice; however, miR-668 inhibition associated with greater renal tubular apoptosis and increased mitochondrial fragmentation. In contrast, augmenting miR-668 activity with a miR-668 mimic diminished mitochondrial fragmentation, decreased renal apoptosis, and mitigated I/R-associated kidney injury.

Deep RNA sequencing (RNA-seq) of miR-induced silencing complexes revealed 680 mRNA species altered in the presence of miR-668, including the gene encoding the protein MTP18. MTP18 levels decreased in renal tissue after $I / R$, and treatment with the miR-668 mimic further reduced MTP18 expression in both sham-operated and I/R-injured mice, further supporting a link between miR668 and MTP18. Knockdown of MTP18 
in cultured renal proximal tubular cells (RPTCs) reduced mitochondrial fragmentation and provided resistance to ATPdepletion-induced apoptosis. Finally, Wei et al. transfected MTP18-knockdown and control RPTCs with miR-668 and found that miR-668 reduced apoptosis in control cells but had no effect on apoptosis in the MTP18-knockdown cells, confirming that miR-668 confers protection in an MTP18-dependent manner.

Wei and colleagues utilized the JASPAR transcription factor-binding profile database to evaluate potential upstream regulators of miR-668 and identified HIF- $1 \alpha$ as a potential candidate. HIF- $1 \alpha$ is a master regulatory transcription factor induced in renal tubules in response to ischemia and hypoxia and is known to upregulate other miRs $(21,22)$. Indeed, proximal tubule-specific deletion of HIF- $1 \alpha$ prevented upregulation of miR688 in kidneys after I/R injury, and chromatin immunoprecipitation assays confirmed HIF- $1 \alpha$ binding to the Mir688 promoter region in cells incubated under hypoxic conditions.

Finally, Wei and colleagues evaluated miR-668 levels in serum from a cohort of hospitalized patients with diverse AKI etiologies and in serum and urine of patients that had undergone cardiopulmonary bypass and aortic cross clamping, a cohort enriched for I/R-associated renal injury. Importantly, this analysis revealed that miR-668 is increased in serum and urine of patients with clinical AKI, suggesting relevance in human disease.

\section{Conclusions and future directions}

The findings of Wei et al. add to our mechanistic understanding of I/R injury in the kidney and should have a marked impact in the field. State-of-the-art molecular technologies were used to reveal the HIF- $1 \alpha$ / miR-668/MTP18 axis as a novel protective mechanism that mitigates mitochondrial fragmentation and kidney injury during I/R. Moreover, Wei et al. were able to confirm upregulation of miR-668 in human patients with AKI, and intriguingly, their study suggests that miR-668 and MTP18 could be potential therapeutic targets for prevention and treatment of diverse etiologies of AKI, not just I/Rassociated disease.
However, further work is needed to validate this pathway in mice and humans with AKI and confirm the role of MTP18 in vivo. Manipulation of miR-668 activity in mice resulted in moderate differences in kidney function but did not change kidney histology at a single time point 48 hours after I/R. Kinetic information on rate of rise, duration, and level of maximal injury, as well as the time to eventual renal recovery in the presence or absence of miR-668 activity may provide important physiologic insights. More robust effects of miR-688 activation were seen in regards to apoptosis, but its effects on other crucial I/R-associated cell death pathways, such as necroptosis, were not evaluated. It also remains to be determined how, and/or if, cell-intrinsic effects of the HIF- $1 \alpha / \mathrm{miR}$ 668/MTP18 axis influence local I/R-induced inflammation.

In humans, despite more than a 2 -fold difference in serum miR-668 expression in the general AKI cohort compared with controls and even greater differences in expression of urinary miR-668 in those with cardiac surgery-associated AKI, these patients still suffered kidney injury. It is possible that extremely high levels of miR- 668 will be needed to achieve pharmacologically enhanced protection from AKI in humans. It remains to be seen whether or not higher expression of miR-668 in patients with AKI correlates with less severe disease in a dose-dependent manner.

Given that Wei and colleagues observed an early rise (within 12 hours) of miR-668 after I/R exposure, therapeutics targeting this pathway may be most efficacious as prophylactics for known highrisk scenarios, such as vascular surgery or transplantation. The identification of HIF$1 \alpha$ as a master regulator of this protective axis is also important, given the development and testing of novel HIF-1 $\alpha$ inhibitors in the cancer field. Knowing the poor history of therapies that have shown benefit in experimental animals but failed in human trials, we must be cautious in our optimism of the HIF- $1 \alpha / \mathrm{miR}-688 / \mathrm{MTP} 18$ axis as a therapeutic target for AKI (23). Further validation will need to be performed in different species of animals and in response to a variety of different types of insults, such as toxin exposure, sepsis, and other multifactorial insults, known to cause human AKI. Still, the findings presented in this article have revealed a potentially targetable pathway in a field that lacks therapeutic options and will help inform our understanding of relevant mechanisms of I/R injury-related diseases.

Address correspondence to: John Cijiang He, Division of Nephrology, Box 1243, Icahn School of Medicine at Mount Sinai, One Gustave L. Levy Place, New York, New York 10029, USA. Phone: 212.659.1703; Email: cijiang.he@mssm.edu.

1. Yellon DM, Hausenloy DJ. Myocardial reperfusion injury. N Engl J Med. 2007;357(11):1121-1135.

2. Bonventre JV, Yang L. Cellular pathophysiology of ischemic acute kidney injury. J Clin Invest. 2011;121(11):4210-4221.

3. Kalogeris T, Baines CP, Krenz M, Korthuis RJ. Cell biology of ischemia/reperfusion injury. Int Rev Cell Mol Biol. 2012;298:229-317.

4. Quiroga I, et al. Major effects of delayed graft function and cold ischaemia time on renal allograft survival. Nephrol Dial Transplant. 2006;21(6):1689-1696.

5. de Perrot M, Liu M, Waddell TK, Keshavjee S. Ischemia-reperfusion-induced lung injury. $\mathrm{Am} \mathrm{J}$ Respir Crit Care Med. 2003;167(4):490-511.

6. Dong Z, Saikumar P, Weinberg JM, Venkatachalam MA. Calcium in cell injury and death. Annu Rev Pathol. 2006;1:405-434.

7. Bonegio R, Lieberthal W. Role of apoptosis in the pathogenesis of acute renal failure. Curr Opin Nephrol Hypertens. 2002;11(3):301-308.

8. Havasi A, Borkan SC. Apoptosis and acute kidney injury. Kidney Int. 2011;80(1):29-40.

9. Linkermann A, et al. Rip1 (receptor-interacting protein kinase 1) mediates necroptosis and contributes to renal ischemia/reperfusion injury. Kidney Int. 2012;81(8):751-761.

10. Iyer SS, et al. Necrotic cells trigger a sterile inflammatory response through the Nlrp3 inflammasome. Proc Natl Acad Sci U S A. 2009;106(48):20388-20393.

11. Chun N, et al. Complement dependence of murine costimulatory blockade-resistant cellular cardiac allograft rejection. Am J Transplant. 2017;17(11):2810-2819.

12. Peng Q, et al. C3a and C5a promote renal ischemia-reperfusion injury. J Am Soc Nephrol. 2012;23(9):1474-1485.

13. Burne MJ, et al. Identification of the CD4(+) $\mathrm{T}$ cell as a major pathogenic factor in ischemic acute renal failure. J Clin Invest. 2001;108(9):1283-1290.

14. Ysebaert DK, et al. T cells as mediators in renal ischemia/reperfusion injury. Kidney Int 2004;66(2):491-496.

15. Uehara M, et al. Ischemia augments alloimmune injury through IL-6-driven CD4. Sci Rep. 2018;8(1):2461.

16. Brooks C, Wei Q, Cho SG, Dong Z. Regulation of mitochondrial dynamics in acute kidney injury in cell culture and rodent models. J Clin Invest. 
2009;119(5):1275-1285.

17. Zhou H, Zhu P, Wang J, Zhu H, Ren J, Chen Y. Pathogenesis of cardiac ischemia reperfusion injury is associated with $\mathrm{CK} 2 \alpha$-disturbed mitochondrial homeostasis via suppression of FUNDC1-related mitophagy. Cell Death Differ. 2018;25(6):1080-1093.

18. Godwin JG, Ge X, Stephan K, Jurisch A, Tullius $S G$, Iacomini J. Identification of a microRNA signature of renal ischemia reperfusion injury. Proc
Natl Acad Sci U S A. 2010;107(32):14339-14344. 19. Wei Q, Bhatt K, He HZ, Mi QS, Haase VH, Dong $Z$. Targeted deletion of Dicer from proximal tubules protects against renal ischemia-reperfusion injury. JAm Soc Nephrol. 2010;21(5):756-761.

20. Wei Q, et al. MicroRNA-668 represses MTP18 to preserve mitochondrial dynamics in ischemic acute kidney injury. JClin Invest. 2018;128(12):5448-5464.

21. Bhatt K, et al. MicroRNA-687 induced by hypoxia- inducible factor- 1 targets phosphatase and tensin homolog in renal ischemia-reperfusion injury. JAm Soc Nephrol. 2015;26(7):1588-1596.

22. Aguado-Fraile E, et al. miR-127 protects proximal tubule cells against ischemia/reperfusion: identification of kinesin family member $3 \mathrm{~B}$ as miR-127 target. PLoS One. 2012;7(9):e44305.

23. de Caestecker $\mathrm{M}$, et al. Bridging translation by improving preclinical study design in AKI. J Am Soc Nephrol. 2015;26(12):2905-2916. 